\title{
The genomic epidemiology of multi-drug resistant nontyphoidal Salmonella causing invasive disease in sub-Saharan Africa
}

Se Eun Park ${ }^{1,2^{*}}$, Duy Thanh Pham ${ }^{3,4 *}$, Gi Deok Pak $^{1}$, Ursula Panzner ${ }^{1}$, Ligia Maria Cruz Espinoza $^{1}$, Vera von Kalckreuth ${ }^{1}$, Justin $\operatorname{Im}^{1}$, Ondari D. Mogeni ${ }^{1}$, Heidi Schütt-Gerowitt ${ }^{1,5}$, John A. Crump ${ }^{6,7,8,9}$, Robert F. Breiman ${ }^{10,11}$, Yaw Adu-Sarkodie ${ }^{12,13}$, Ellis Owusu-Dabo ${ }^{12,13}$, Raphaël Rakotozandrindrainy $^{14}$, Abdramane Bassiahi Soura ${ }^{15}$, Abraham Aseffa ${ }^{16}$, Nagla Gasmelseed ${ }^{17,18}$, Arvinda Sooka ${ }^{19}$, Karen H. Keddy ${ }^{20}$, Jürgen May ${ }^{21,22}$, Peter Aaby ${ }^{23,24}$, Holly M. Biggs ${ }^{6,7}$, Julian T.

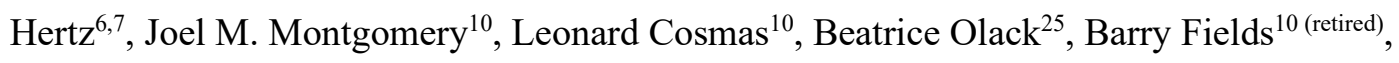
Nimako Sarpong ${ }^{12,22}$, Tsiriniaina Jean Luco Razafindrabe ${ }^{14}$, Tiana Mirana Raminosoa ${ }^{14}$, Leon Parfait Kabore ${ }^{26}$, Emmanuel Sampo ${ }^{26}$, Mekonnen Teferi ${ }^{16}$, Biruk Yeshitela ${ }^{16}$, Muna Ahmed El Tayeb $^{17}$, Ralf Krumkamp ${ }^{21,22}$, Denise Myriam Dekker ${ }^{21,22}$, Anna Jaeger ${ }^{21,22}$, Adama Tall ${ }^{27}$, Aissatou Niang ${ }^{27}$, Morten Bjerregaard-Andersen ${ }^{23,24}$, Sandra Valborg Løfberg ${ }^{23,24}$, Jessica Fung Deerin $^{1}$, Jin Kyung Park ${ }^{1}$, Frank Konings ${ }^{1}$, Sandra Van Puyvelde ${ }^{28,29,30}$, Mohammad Ali1 ${ }^{1,31}$, John D. Clemens ${ }^{1,32,33}$, Gordon Dougan ${ }^{30}$, Stephen Baker $^{30 \S}$ and Florian Marks $s^{1,30 \S}$

${ }^{1}$ International Vaccine Institute, SNU Research Park, 1 Gwanak-ro, 1 Gwanak-gu, Seoul, 08826, Republic of Korea

${ }^{2}$ Graduate School of Public Health, Yonsei University, 50 Yonsei-ro, Seodaemun-gu, Seoul, 03722, Republic of Korea

${ }^{3}$ The Hospital for Tropical Diseases, Wellcome Trust Major Overseas Programme, Oxford University Clinical Research Unit, 764 Vo Van Kiet, Quant 5, Ho Chi Minh City, Vietnam

${ }^{4}$ Centre for Tropical Medicine and Global Health, University of Oxford, Old Road campus, Roosevelt Drive, Headington, Oxford OX3 7FZ, United Kingdom

${ }^{5}$ Institute of Medical Microbiology, University of Cologne, 50923 Cologne, Germany

${ }^{6}$ Kilimanjaro Christian Medical Centre, PO Box 3010, Moshi, Tanzania

${ }^{7}$ Division of Infectious Diseases and International Health, Duke University Medical Center, 
Durham, NC 27710, USA

${ }^{8}$ Duke Global Health Institute, Duke University, Durham, NC 27708, USA

${ }^{9}$ Centre for International Health, University of Otago, Dunedin 9054, New Zealand

${ }^{10}$ Centers for Disease Control and Prevention, KEMRI Complex, Mbagathi Road off Mbagathi Way, PO Box 606-00621, Village Market, Nairobi, Kenya

${ }^{11}$ Global Health Institute, Emory University, 201 Dowman Dr, Atlanta, Georgia, 30322 USA

${ }^{12}$ Kwame Nkrumah University of Science and Technology, PO Box PMB KNUST, Kumasi, Ghana

${ }^{13}$ Kumasi Centre for Collaborative Research in Tropical Medicine, Kwame Nkrumah University of Science and Technology, KCCR, UPO, PMB, KNUST, Kumasi, Ghana

${ }^{14}$ University of Antananarivo, BP 566, Antananarivo 101, Madagascar

${ }^{15}$ Institut Supérieur des Sciences de la Population, University of Ouagadougou, 03 BP 7118, Ouagadougou 03, Burkina Faso

${ }^{16}$ Armauer Hansen Research Institute, Jimma Road, ALERT Compound, PO Box 1005, Addis Ababa, Ethiopia

${ }^{17}$ Faculty of Medicine, University of Gezira, PO Box 20, Wad Medani, Sudan

${ }^{18}$ Faculty of Science, University of Hafr Al Batin, Al Jamiah, Hafr Albatin 39524, Saudi Arabia

${ }^{19}$ National Institute for Communicable Diseases, 1 Modderfontein Road, Sandringham,

Johannesburg, South Africa

${ }^{20}$ School of Public Health, Faculty of Health Sciences, University of the Witwatersrand, 1 Jan Smuts Avenue, Braamfontein 2000, Johannesburg, South Africa

${ }^{21}$ Bernhard Nocht Institute for Tropical Medicine, Bernhard Nocht Str. 74, 20359, Hamburg, Germany

${ }^{22}$ German Center for Infection Research, Hamburg- Lübeck-Borstel-Riems, Inhoffenstrabe 7, 38124 Braunschweig, Germany

${ }^{23}$ Bandim Health Project, Apartado 861, 1004 Bissau codex, Bissau, Guinea-Bissau

${ }^{24}$ Research Center for Vitamins and Vaccines, Bandim Health Project, Statens Serum Institut, 5 
Artillerivej, DK-2300, Copenhagen, Denmark

${ }^{25}$ Kenya Medical Research Institute, Mbagathi Rd. Nairobi, Kenya PO BOX 54840-00200

${ }^{26}$ Schiphra Hospital, Ouagadougou, Burkina Faso

${ }^{27}$ Institute Pasteur de Dakar, 36 Avenue Pasteur, B.P. 220, Dakar, Senegal

${ }^{28}$ Department of Biomedical Sciences, Institute of Tropical Medicine, Antwerp, Belgium

${ }^{29}$ Laboratory of Medical Microbiology, Vaccine \& Infectious Disease Institute, University of Antwerp, Antwerp, Belgium

${ }^{30}$ Cambridge Institute of Therapeutic Immunology \& Infectious Disease (CITIID), Department of Medicine, University of Cambridge, Box 157, Hills Road, Cambridge, CB2 0QQ, United Kingdom

${ }^{31}$ Johns Hopkins Bloomberg School of Public Health, 615 N Wolfe St., Baltimore, Maryland, 21205 USA

${ }^{32}$ International Centre for Diarrheal Disease Research, Bangladesh, GPO Box 128, Dhaka 1000, Bangladesh

${ }^{33}$ University of California, Fielding School of Public Health, 650 Charles E. Young Dr. South, Center for Health Sciences, Los Angeles, CA 90095-1772, USA

${ }^{*}$, Contributed equally

${ }^{\#}$ Corresponding author:

Se Eun Park, PhD; International Vaccine Institute, 1 Gwanak-ro, 1 Gwanak-gu, Seoul, 151-742;

Republic of Korea; T: +82-2-881-1154; Email: SeEun.Park@ivi.int 


\begin{abstract}
Background: Invasive nontyphoidal Salmonella (iNTS) is one of the leading causes of bacteraemia in sub-Saharan Africa. Multi-drug resistance (MDR) and further resistance to third generation cephalosporins and fluoroquinolones have emerged in multiple iNTS serotypes. Molecular epidemiological investigations of nontyphoidal Salmonella are needed to better understand the genetic characteristics and transmission dynamics associated with major MDR iNTS serotypes across the continent.
\end{abstract}

Methods: A total of 166 nontyphoidal Salmonella isolates causing invasive disease were collected from a multi-centre study in eight African countries between 2010 and 2014, and whole-genome sequenced to investigate the geographical distribution, antimicrobial genetic determinants and population structure of iNTS serotypes-genotypes. Phylogeographical reconstruction was further conducted in context of the existing genomic framework of iNTS serotypes Typhimurium and Enteritidis. Population-based incidence of MDR-iNTS disease was also estimated.

Results: Salmonella enterica subsp. Enterica serotype Typhimurium (S. Typhimurium) sequence-type (ST) 313 and Salmonella enterica subsp. Enterica serotype Enteritidis (S. Enteritidis) ST11 were predominant, and both exhibited high frequencies of MDR. Salmonella enterica subsp. Enterica serotype Dublin (S. Dublin) ST10 emerged in West Africa. Mutations in the gyrA gene were identified in $S$. Enteritidis and $S$. Typhimurium in Ghana; and ST313 carrying $b l a_{\mathrm{CTX}-\mathrm{M}-15}$ was found in Kenya. Inter-country transmission of MDR ST313 lineage II and the West African Clade of MDR ST11 between Ghana and neighbouring countries including Mali, Burkina Faso, and Nigeria were evident. The incidence of MDR-iNTS disease exceeded 100/100,000-person years-of-observation (PYO) in children aged $<5$ years in several West African countries. 
Conclusions: Multiple MDR iNTS serotypes-sequence types, predominantly $S$. Typhimurium ST313 and S. Enteritidis ST11, are co-circulating in sub-Saharan Africa with evidence of transmission between West African countries. The development of safe and effective iNTS vaccines coupled with appropriate antimicrobial stewardship and adequate epidemiological monitoring are essential to limit the impact of these pathogens in Africa.

\section{Keywords}

Invasive nontyphoidal Salmonella (iNTS); multi-drug resistance; antimicrobial resistance; MDR iNTS disease incidence; genotypes; phylogeny; phylogeography; genomics; Africa; iNTS vaccines 


\section{Background}

Non-typhoidal Salmonella enterica are classically considered a collective of zoonotic pathogens associated with self-limiting diarrhoea in humans. However, certain nontyphoidal Salmonella serovars have become an established cause of invasive disease (iNTS) in specific geographical regions, particularly affecting immunocompromised individuals including infants and young adults with HIV, malaria and malnutrition [1]. Annually, there are an estimated 3.4 million cases of iNTS disease globally, $20 \%$ of these are fatal [2]'[3]. The vast majority of iNTS diseases occur in sub-Saharan African countries where annual incidences up to $175-388$ per 100,000 person-years and case fatality ratio (CFR) as high as $25 \%$ have been reported in children aged $<5$ years [4],[5],[6],[7],[8]. A recent multicentre study across sub-Saharan Africa also reported iNTS as a major cause of bacteraemia in febrile patients in Africa, with adjusted incidences $>100$ per 100,000 person-years identified across multiple sampling locations [9].

Various Salmonella serovars can cause iNTS disease; however, previous studies have shown that the majority of iNTS infections in sub-Saharan Africa are caused by two predominant serovars, namely Salmonella enterica subsp. Enterica serotype Typhimurium (S. Typhimurium) and Salmonella enterica subsp. Enterica serotype Enteritidis (S. Enteritidis) [10],[11],[12],[13]. $S$. Typhimurium in sub-Saharan Africa are largely associated with a single multi-drug resistant (MDR) genotype, known as ST313, comprised of two major lineages I and II [14]. S. Enteritidis are estimated to account for a third of the iNTS burden in sub-Saharan Africa and are primarily associated with the ST11 genotype. Further, there are three major clades of S. Enteritidis ST11 (Global epidemic, West African and Central/East African clades) co-circulating in this region with a rapid increase in MDR [15]. In addition to $S$. Typhimurium and $S$. Enteritidis, other Salmonella enterica subsp. Enterica serovars associated with iNTS disease include $S$. Isangi [16], S. Concord [17], S. Stanleyville, and S. Dublin [18], signifying the complex epidemiological landscape of iNTS in sub-Saharan Africa. 
Historically, ampicillin, chloramphenicol, and trimethoprim-sulfamethoxazole (co-trimoxazole) have been the first-line drugs for the treatments of iNTS disease as well as typhoid fever in Africa [19]. The increased use of antimicrobials has consequently led to the emergence and widespread of MDR, defined as resistant to these first-line drugs, in iNTS organisms; particularly $S$. Typhimurium and $S$. Enteritidis [10][20]. Numerous sub-Saharan African countries have reported high prevalences of MDR iNTS disease, including Malawi [20-22], Kenya [23]:[24]'[25], Ghana [26], Gambia [5], Democratic Republic of the Congo (DRC) [27], Mozambique [7,28], Tanzania [29], Burkina Faso [9], Guinea-Bissau [9], and Nigeria [30]. Consequently, alternative treatments, such as ciprofloxacin, azithromycin, and ceftriaxone, are being increasingly deployed to manage such infections. However, these drugs are unavailable or costly in some resource-limited settings [13],[22]. Their effectiveness is likely diminished further due to the emergence of MDR plus ceftriaxone resistance as well as extensively drugresistance (XDR), defined as MDR combined with resistance to fluoroquinolones (i.e. ciprofloxacin) and third generation cephalosporins (i.e. ceftriaxone), as reported in $S$. Typhimurium ST313 in Kenya [19], Malawi [12], and the DRC [31]. The continental dissemination of MDR organisms together with the emergence of highly resistant variants pose a significant challenge for the treatment, management, and control of iNTS disease in Africa [19].

Here, through whole genome sequencing (WGS), we aimed to investigate the distribution of iNTS serovars and their corresponding sequence types (ST) and antimicrobial resistance (AMR) determinants through a contemporaneous collection of iNTS strains from multiple sites across sub-Saharan Africa. We additionally estimated the incidence of MDR iNTS disease in the sampling locations and performed comprehensive phylogeographical analyses of $S$. Typhimurium ST313 and S. Enteritidis ST11 in the context of globally representative collection of respective organisms. 


\section{Methods}

Study design and inclusion criteria

Between 2010 and 2014, standardized healthcare facility-based surveillance of invasive Salmonella infections in sub-Saharan African populations was conducted in thirteen sites in ten countries (Typhoid Fever Surveillance in Africa Program, TSAP); the methods were published [32]. In brief, febrile patients from all age groups (except in Ghana, where only children aged $<15$ years were enrolled) with a tympanic or axillary temperature of $\geq 38.0^{\circ} \mathrm{C}$ or $\geq 37.5^{\circ} \mathrm{C}$, respectively, living in a defined study catchment area were eligible for recruitment. For inpatients, reported fever within the period of 72 hours prior to admission was added to the inclusion criteria. Written informed consent/assent was obtained. Clinical assessments of patients included history and current of illness, physical examination, and clinical appraisal. Eligible patients were treated per the respective national guidelines. Blood samples $(5-10 \mathrm{~mL}$ for adults; 1-3 mL for children) were collected for laboratory diagnostics of these febrile cases.

\section{Bacterial isolates and antimicrobial susceptibility testing}

Through the TSAP study, blood specimens were obtained from febrile patients; one aerobic blood culture bottle per patient. Collected bottles were incubated in systems with automated growth detection (BACTEC Peds Plus Medium/BACTECT Plus Aerobic-F, BACTEC, BectonDickinson, New Jersey; or BacT/ALERT PF Paediatric FAN/BacT/ALERT FA FAN Aerobic, bioMerieux, Marcy l'Etoile, France) except for the study site in Sudan whereby manual blood culture techniques were used. Blood cultures with bacterial growth were sub-cultured on solid media such as blood agar and chocolate agar (Oxoid, Basingstoke, United Kingdom), and biochemical tests were conducted (API 20E, bioMerieux) to confirm suspected Salmonella isolates [32]. Confirmatory testing was conducted at the study reference laboratories at Oxford University Clinical Research Unit (OUCRU, Ho Chi Minh city) and Bernhard Nocht Institute for Tropical Medicine (BNITM) using the VITEK automate. Antimicrobial susceptibility testing was performed using agar diffusion tests according to the Clinical Laboratory and Standards 
Institute (CLSI) guidelines [32]. Malaria diagnostics were performed via a combination of thick and thin blood smears and rapid malaria test (SD Bioline Malaria Ag P.f./P.v., SD Standard Diagnostics, Suwon, Republic of Korea), this was variable between sites [33].

\section{Data sources and bacterial isolates}

The total of 166 iNTS isolates was used for this investigation, which comprised 94 iNTS isolates from the TSAP study, an additional 23 iNTS isolates detected outside the predefined study catchment area, and 49 iNTS isolates from other studies conducted in Agogo, Ghana in 2007-2009. For further analyses on the $S$. Typhimurium and $S$. Enteritidis isolates in context of the global phylogeny of respective organisms, existing datasets were incorporated: 102 iNTS serovar Typhimurium ST313 Lineage II isolates from seven countries (Malawi, Kenya, Mozambique, Uganda, DRC, Nigeria, and Mali) [10], Nigeria and DRC [11], Malawi [12], Kenya [13], Malawi [34], and 594 iNTS serovar Enteritidis ST11 isolates (selected from Feasey et al. 2016) [15]. A summary of these isolates and their country of origin is provided in Supplementary Table 1.

\section{Whole genome sequencing}

Genomic DNA was extracted from all Salmonella isolates using the Wizard Genomic DNA Extraction Kit (Promega, Wisconsin, USA). Two $\mu \mathrm{g}$ of genomic DNA from each organism was subjected to indexed-tagged pair-end sequencing on an Illumina Hiseq 2000 platform (Illumina, CA, USA) to generate 100 bp paired-end reads. Raw sequence data are available in the European Nucleotide Archive (Project number: ERP009684, ERP010763, ERP013866).

\section{Single Nucleotide Polymorphism (SNP) calling and analyses}

Raw Illumina reads were used to create multiple assemblies using Velvet v1.2 [35] with parameters optimized using VelvetOptimiser v2.2.5 [36][37] and automated annotation was performed using PROKKA v1.5 [38]. Roary [39] was used to define the pan genome of 166 
iNTS isolates with blastp percentage identity of $99 \%$ and a core definition of $99 \% .3,450$ core genes were identified (genes that present in $\geq 99 \%$ strains) and 86,765 SNP sites were extracted from the core gene alignment using SNP-sites [37].

For $S$. Typhimurium ST313, raw Illumina reads of 99 isolates from this study and additional 102 isolates of $S$. Typhimurium ST313 lineage II from previous studies [10],[11],[12], [13],[34] were mapped to the reference sequence of $S$. Typhimurium strain SL1344 (accession: FQ312003.1), using SMALT version 0.7.4 (http://www.sanger.ac.uk/resources/software/smalt/). Candidate SNPs were called against the reference sequence using SAMtools [40] and filtered with a minimum mapping quality of 30 and minimum consensus base agreement of $75 \%$. The allele at each locus in each isolate was determined by reference to the consensus base in that genome using SAMtools mpileup and removing low confidence alleles with consensus base quality $\leq 20$, read depth $\leq 5$ or a heterozygous base call. Repeat finding program in NUCmer v3.1 [41] was used to identify exact repetitive regions of $\geq 20 \mathrm{bp}$ in length in the reference genome and SNPs called in these regions were excluded. SNPs called in phage sequences and recombinant regions identified using Gubbins [42] were further removed, resulting in a final set of 1,960 chromosomal SNPs. The identification of SNPs for S. Enteritidis ST11 was performed following the same procedure as $S$. Typhimurium ST313. Briefly, the raw Illumina reads of $28 S$. Enteritidis ST11 isolates from my studies and additional 594 isolates from a global collection [15] were mapped to the reference sequence of $S$. Enteritidis strain P125109 (accession: NC_011294.1), using SMALT followed by SNP calling and filtering as above resulting in a final set of 25,121 SNPs.

\section{Phylogenetic analyses}

A maximum likelihood (ML) phylogenetic tree was constructed from the 86,765 SNP alignment of all 166 iNTS isolates using RAxML version 8.2.8 with a generalized time-reversible model and a Gamma distribution to model the site-specific rate variation (GTRGAMMA in RAxML) 
[43], and outgroup rooted. Clade support for this tree was assessed through a bootstrap analysis with 100 pseudo-replicates. To investigate the molecular epidemiology of my $S$. Typhimurium ST313 and S. Enteritidis ST11 isolates in regional and international context, ML tree was inferred from an alignment of 1,960 SNPs for $201 S$. Typhimurium ST313 lineage II isolates (99 from this study and 102 from previous studies [10],[11],[12],[13];[34] and an alignment of 25,121 SNPs for $622 S$. Enteritidis ST11 isolates (28 from this study and 594 from a global collection [15], using RAxML with the same parameters as above. Support for these phylogenetic trees was assessed through a 100 bootstrap pseudo-analysis. Tree annotation was visualized using ITOL [44].

\section{Antimicrobial resistant gene and plasmid analyses}

From raw Illumina reads, Short Read Sequence Typer- SRST2 [45] was used to identify the acquired AMR genes and their precise alleles using the ARG-Annot database [46], as well as the plasmid replicons using the PlasmidFinder database [47]. Multilocus sequence typing (MLST) of all iNTS isolates was also determined using SRST2 together with MLST database for Salmonella enterica downloaded from pubMLST. Salmonella serovars were identified using conventional serology as well as MLST [48] and genomics [49]. To investigate the isolates with resistant genotypes, a de novo genome assembly was performed from raw sequences using SPAdes [50] version 3.11.0, followed by Bandage [51] to inspect the location of AMR genes in the corresponding assembled contigs.

\section{Incidence analyses of MDR iNTS disease}

Incidence of MDR iNTS was estimated per 100,000-person years-of-observation (PYO) for MDR iNTS isolates detected from Burkina Faso, Ghana, Guinea-Bissau, Kenya, and Senegal. Statistical methodology used previously to calculate the incidence of $S$. Typhi and iNTS disease in TSAP countries was used to calculate MDR iNTS incidence [9],[32], [52]. Briefly, an agestratified PYO were estimated using available demographic data in HDSS (Health and 
Demographic Surveillance System) and non-HDSS sites and health-seeking behaviour of randomly selected individuals, representative of the study population, were factored in (denominator). The recruitment proportion was adjusted to the age-stratified crude MDR iNTS cases (numerator). Adjusted incidence of MDR iNTS per 100,000 PYO was estimated with 95\% CIs using these adjustment factors and crude MDR iNTS case numbers. The previously established multi-country database (FoxPro software) for TSAP was used for the countries with MDR iNTS isolates.

\section{Results}

The geographical distribution of iNTS serovars and sequence types in Africa

The majority of iNTS infections in sampled sub-Saharan African countries were caused by two predominant serovars-STs, $S$. Typhimurium ST313 and S. Enteritidis ST11; however, their distribution varied between countries (Table 1). More specifically, 66\% (110/166) of the isolates were $S$. Typhimurium, of which 90\% (99/110) were ST313 and 10\% (11/110) ST19. S.

Enteritidis accounted for 18\% (30/166) of the isolates, comprising ST11 (93.3\%; 28/30), ST183 $(3.3 \% ; 1 / 30)$ and ST2107 (3.3\%; 1/30). Notably, S. Dublin (ST10) also accounted for $11 \%$ $(18 / 166)$ of the iNTS isolates. We also found several less common serovars-STs including $S$. Choleraesuis ST145 (3/166), S. Muenster ST321 (1/166), S. Poona ST308 (1/166), $S$. Stanleyville ST339 (1/166), and S. Virchow ST359 (1/166).

Regarding the geographical distribution of iNTS, S. Typhimurium ST313 were predominately found in West Africa (Burkina Faso, Ghana, Guinea-Bissau). In East and Southern Africa, ST313 was exclusively detected in Kenya (Table 1). S. Enteritidis ST11, though less common than ST313, appeared to be pervasive in both West (Burkina Faso, Ghana, Guinea-Bissau, Senegal) and Southern (South Africa, Madagascar) Africa. Additionally, S. Dublin ST10 was only detected in Burkina Faso and Ghana in West Africa. S. Typhimurium ST19 appeared geographically dispersed across the continent, including West (Burkina Faso, Ghana, Guinea- 
Bissau, Senegal) and East and Southern Africa (Tanzania, Madagascar).

Overall, 61\% (102/166) of the iNTS organisms were multi-drug resistant. MDR iNTS organisms were isolated in Burkina Faso (83\%; 10/12), Ghana (66\%, 88/133), Guinea-Bissau $(22 \% ; 2 / 9)$, Kenya $(100 \% ; 1 / 1)$, and Senegal $(50 \% ; 1 / 2) . S$. Typhimurium exhibited the highest prevalence of MDR (85\%; 94/110), and notably 95\% (94/99) of the ST313 isolates were MDR. $23 \%(7 / 30)$ of $S$. Enteritidis and 6\% (1/18) S. Dublin were MDR. None of the $11 S$. Typhimurium ST19 isolates were MDR (Figure 1, Table 2).

\section{Phylogenetics and AMR determinants of iNTS serovars}

Our phylogenetic reconstruction of all iNTS isolates showed that the three major serovars-STs: $S$. Typhimurium (ST313), S. Enteritidis (ST11), and S. Dublin (ST10) formed three distinct clusters and exhibited dissimilar AMR gene profiles (Figure 2). Almost all of the MDR $S$. Typhimurium ST313 (95\%; 94/99) carried the Tn21 transposon-associated MDR-loci (sulIIstrAB-dfrAl-aadA1-sulI-cat-blaTEM) on an IncF virulent-resistance plasmid pSLT-BT as reported before [10]. One MDR S. Typhimurium ST313 from Kenya additionally carried two copies of $b l a_{\mathrm{CTX-M-15}}$, conferring resistance to third generation cephalosporins, one of which was located on the 300kb IncHI2 plasmid, pKST313 (accession number: LN794248). The other bla $a_{\mathrm{CTX}-\mathrm{M}-15}$ was inserted into the chromosome and disrupted the ompD locus. Of $99 \mathrm{~S}$. Typhimurium ST313, 5 (5.1\%) harboured pSLT-BT, but were not associated with MDR due to the lack of MDR-loci. Reduced suseptibility to fluoroquinolones was uncommon in $S$. Typhimurium ST313, with 2\% (2/99) of Ghanaian ST313 isolates possessing a mutation (S87Y) in $g y r A$ (Table 2).

The majority of $S$. Enteritidis ST11 (19/28; 68\%) harboured a typical IncF virulence plasmid (60kb), which was comparable to pSENV (accession number NC_019120.1, coverage 100\%, identity 99\%). The remaining S. Enteritidis ST11 (9/28; 32\%) harboured a novel IncI1 
virulence-resistance plasmid (pSEP, accession number: ERP121368) of approximately 68kb (Figure 3), of which 7/28 (25\%) isolates (4/Burkina Faso, 2/Ghana, 1/Senegal) carried the MDR-encoding Tn21-like transposon (sulII-strAB-tetB-dfrA1-sulI-cat-blaTEM), and 2/28 (7.1\%) isolates carried an AMR cassette (TnpA-sulII-strAB-blaTEM-Tn3), conferring resistance against ampicillin, streptomycin, and sulphanomides. The novel IncI plasmid exhibited $60 \%$ homology to pSENV and did not harbour the IncF replicons, the pefBACD fimbriae-encoding operon, or the virulent-associated genes $\operatorname{srg} A$ and $r c k$ (Figure 3). In addition, two non-MDR Ghanaian S. Enteritidis ST11 possessed an AMR cassette (sul2-strAB-tetA) associated with a small (11kb) non-conjugative IncQ plasmid conferring resistance against sulphonamides, streptomycin and tetracyclines. This IncQ plasmid exhibited a similar genetic structure to pSTU288-2 from $S$. Typhimurium (accession number CP004059.1, coverage 98\%, identity 99\%). Two further non-MDR Ghanaian isolates carried a Tn21-mediated AMR cassette (sulIIstrAB-dfrA1-aadA1-sulI-cat) on the virulence plasmid, and a single non-MDR isolate from Madagascar carried a blaTEM-bearing Tn3 integrated into the virulence plasmid. Reduced suseptibility to fluoroquinolones was predicted in 39\% (11/28) of the S. Enteritidis-ST11, all of which originated from Ghana, displaying differing mutations in codon 87 in gyrA (D87G: 6 isolates, D87N: 3 isolates, and D87Y: 2 isolates) (Table 2).

Most $S$. Dublin ST10 isolates (17/18; 94\%) did not carry any AMR genes, except for one Ghanaian isolate with an MDR-encoding Tn21 transposon-mediated cassette (sulII-strABdfrA1-aadA1-sulI-cat-blaTEM) (Figure 2). All S. Dublin ST10 isolates carried a typical IncF virulence plasmid (75kb), similar to pOU1115 (accession number: NC_010422.1, coverage $100 \%$, identity 99\%). None of the S. Dublin ST10 isolates possessed mutations in gyrA (Table 2). Markedly, the viaB operon encoding the Vi polysaccharide was absent from all S. Dublin isolates. Lastly, all S. Typhimurium ST19, as well as other uncommon serovars-STs, had no acquired AMR genes and considered pan-antimicrobial susceptible. 
Phylogeography of S. Typhimurium ST313 and S. Enteritidis ST11 isolates in global context To investigate the potential transmission patterns of predominant iNTS serovars of $S$. Typhimurium ST313 and $S$. Enteritidis ST11 in a broader context, we reconstructed the phylogenetics of our isolates together with previously published data. We found that all $S$. Typhimurium ST313 isolated from our study belong to lineage II, suggesting lineage I may no longer be circulating in the sampled countries. One Kenyan S. Typhimurium ST313 with two copies of $b l a_{\mathrm{CTX}-\mathrm{M}-15}$ formed part of the previously described clonal expansion of the MDR ceftriaxone-resistant ST313 sublineage in Kenya [19]. The ST313 from Ghana displayed a high degree of genetic diversity and scattered around the phylogenetic tree. This observation indicates these organisms have been likely circulating in Ghana for a prolonged period. The ST313 from Ghana clustered with isolates from Mali, Burkina Faso, and Nigeria on several occasions, indicating frequent movement of organisms between Ghana and the neighbouring West African countries (Figure 4a).

Further phylogeographical investigation of $S$. Enteritidis ST11 in a global context demonstrated that, 11/28 (40\%) (Ghana: 5, Burkina Faso: 4, Senegal: 1, Guinea Bissau: 1) isolates fell into the West African clade described by Feasey et al, known to be associated with AMR and an invasive disease phenotype[15]. Our ST11 organisms within this West African clade also displayed an MDR (7 isolates) or AMR phenotype ( 2 isolates). Within this clade, we found evidence that some Ghanaian isolates clustered tightly with isolates from Burkina Faso and previously described isolates from Mali. Additionally, 13/28 (46\%) of the $S$. Enteritidis ST11 (Ghana 11, Madagascar: 1, South Africa: 1) belonged to the Global epidemic clade described by existing publication, of which these isolates appeared as part of their country-specific endemic circulation; however, we also found that some Ghanaian isolates clustered with previously described isolates from its neighbouring Cameroon and Senegal. We identified 4/28 (14\%) of the ST11 isolates grouped within the Outlier cluster, of which 2 Ghanaian isolates and two isolates from Madagascar clustered with previously described isolates from the U.S. and 
Mauritius, respectively. All these organisms were susceptible to all antimicrobials (Figure 4b).

\section{Incidence of MDR iNTS disease in sub-Saharan Africa}

The age stratified incidences of MDR iNTS in Burkina Faso, Ghana, Guinea-Bissau, and Kenya are illustrated in Table 3. The adjusted incidence of MDR iNTS disease exceeded 100/100,000person years-of-observation (PYO) in children $<15$ years of age in all West African countries: Burkina Faso (NiokoII, 274/100,000 PYO, 95\% confidence interval [CI], 185-406; and Polesgo, 255/100,000 PYO, 95\% CI, 138-470), Ghana (Asante Akim North: Ghana-AAN, 414/100,000, 95\% CI, 333-515), and Guinea-Bissau (Bandim, 105/100,000, 95\% CI, 69-161). Among children $<15$ years, younger children $(<2-4$ years) exhibited the highest MDR iNTS incidences in both sites in Burkina Faso: 753/100,000 PYO (95\% CI, 460-1233) in NiokoII and 630/100,000 PYO (95\% CI, 288-1380) in Polesgo. In both settings in Burkina Faso, the incidence of MDR iNTS disease in the infant age-group was slightly lower than in the 2-4-year olds though all children $<5$ years exhibited a high burden of MDR iNTS disease. In GhanaAAN, infants aged $<2$ year had the highest incidence of MDR iNTS disease $(1435 / 100,000 ; 95 \%$ CI, 1110-1854) followed by children aged between 2 and 5 years (747/100,000; 95\% CI, 4911135). Similarly in Guinea-Bissau, infants $<2$ years exhibited the highest incidence of MDR iNTS disease (291/100,000; 95\% CI, 176-482). The incidence rate of MDR iNTS in older age groups ( $\geq 15$ years) was relatively low, ranging between 0 (Guinea-Bissau), to 11 (Kenya), and 54 (Burkina Faso) per 100,000 PYO.

\section{Discussion}

Although much understanding about iNTS disease in Africa has been gained over the last decade, the disease remains a major public health problem in parts of sub-Saharan Africa. While there is currently no vaccine for iNTS disease, the disease burden is further exacerbated by the regional dissemination of MDR phenotypes and the emergence of resistance against newer drugs in some countries. Moreover, iNTS disease has been caused by a large number of 
Salmonella serovars with indistinguishable clinical symptoms [2]. NTS serovars may exhibit different propensity to develop drug resistance and have dissimilar host range and transmission patterns [13],[53]. It is therefore critical to monitor the distribution of iNTS serovars and their AMR patterns over time and across the continent to facilitate updated treatment guidelines for local public health authorities and healthcare workers.

Our study found high frequency of MDR iNTS infections in the sampled countries in subSaharan Africa. Despite the fact that MDR S. Typhimurium ST313 remains the most common cause of iNTS disease, our data highlight the emergence of other less common MDR iNTS serovars; particularly MDR S. Enteritidis ST11 and S. Dublin ST10 in West Africa. Recent studies have also reported that MDR S. Enteritidis ST11 appears to be an emerging cause of bloodstream infections in Africa [54][55]. The source and predominant modes of transmission for these increasingly important iNTS serovars are currently unknown and requires further investigation. In addition, the co-circulation of multiple MDR iNTS serovars poses a substantial challenge for the control and prevention measures. Vaccines for iNTS disease need to target multiple key Salmonella serotypes and such efforts are currently underway including a bivalent O-antigen polysaccharide-CRM197 conjugate vaccine targeting both $S$. Typhimurium and $S$. Enteritidis, using Generalized Modules for Membrane Antigens (GMMA) technology [56] and an iNTS vaccine program by the International Vaccine Institute (IVI) [57].

Typically, iNTS serovars possess a non-conjugative virulence plasmid of IncF replicon type; as a result, the acquisition of the novel virulence-resistance IncI1 plasmid carrying both the $s p v$ locus and MDR-encoding cassette in S. Enteritidis ST11 is worrying as such plasmid may be further disseminated via horizontal gene transfer. This phenomenon also raises the possibility that different iNTS serovars may have access to different resistance gene pools and may have capacities to increase virulence and/or resistance. Routine surveillance with the integration of WGS should be implemented to closely monitor the trend in antimicrobial resistance and 
dominant iNTS serovars as well as to timely detect novel AMR clones that are epidemiologically and clinically important for informing public health and clinical practices.

Our reconstructed phylogeography of the two predominant MDR serovars-genotypes $(S$. Typhimurium ST313 and $S$. Enteritidis ST11) indicated all contemporaneous $S$. Typhimurium ST313 fell into lineage II with evidence of occasional transfer between Ghana and the neighbouring countries (Burkina Faso, Guinea-Bissau, Senegal, and Nigeria) in West Africa. The ST11 isolates belonged to three distinct lineages (Global epidemic clade, West African clade and an Outlier cluster), of which all MDR ST11 isolates fell within the West Africa clade and appeared to be widely distributed throughout Ghana, Burkina Faso, and Senegal. The observation that multiple ST11 lineages are found to cause bloodstream infections is worrying as these organisms are associated with multiple transmission pathways and may have the ability to acquire additional virulence-resistance plasmids [58]. Previous studies suggest that asymptomatic carriers are likely an important reservoir of nontyphoidal Salmonella [59],[60], which may contribute to the transmission of these pathogens within and between countries. Given the complex transmission mechanisms of iNTS/NTS serovars, one health genomic approach should be also considered in an attempt to better understand the sources and transmission routes of various iNTS/NTS serovars across different ecological niches. Further, understanding population mobility across the continent will contribute to monitoring disease expansion and the success of local efforts at control.

Antimicrobial therapy is essential to treat patients with iNTS disease and to reduce mortality. However, the effectiveness of antimicrobial therapy is diminishing due to the emergence and dissemination of various MDR and XDR NTS serovars and strains [15],[31],[61]. Our data provides further insights into the emergence of reduced susceptibility to fluoroquinolones among MDR ST313 and ST11 from Ghana as well as the continued circulation of ceftriaxoneresistant ST313 sublineage in Kenya. Invasive Salmonella with reduced susceptibility to 
ciprofloxacin have also been increasingly reported in Burkina Faso [9], Ghana [9,26], Nigeria [62], Senegal [63], Mozambique [28], the Congo [64], and South Africa [65]. This increasing trend in AMR against clinically important classes of newer antimicrobials among multiple iNTS serovars in Africa is of particular concern given the fact that patients infected with highly resistant organisms are likely not to respond to treatment with these newer drugs [19]. It is probable that the widespread use of ceftriaxone and ciprofloxacin for the treatment of febrile illnesses in Africa will eventually lead to a rapid increase and spread of MDR and XDR pathogens in this continent, which may amplify the burden of the disease.

We also found a substantial incidence of MDR iNTS disease in the West African countries including Burkina Faso, Ghana ( $<5$ years), and Guinea-Bissau ( $<2$ years). The incidence of MDR iNTS disease presented here generally corresponded to the incidence of iNTS disease in respective countries [9]. Among these Western African countries, Ghana in particular exhibited high incidences of both MDR iNTS disease and MDR typhoid fever reported previously [66], indicative of concurrence of iNTS disease and typhoid fever in Ghana with increasing public health concern associated with invasive MDR salmonellosis. Conversely, in East African countries such as Kenya and Tanzania, MDR iNTS was less prominent relative to the high incidence of MDR typhoid fever in some of the same locations [66].

Our study did not take into account the prior usage of antimicrobials nor other clinical features reported with iNTS disease such as gastrointestinal symptoms [67], and our enrolment criteria excluded afebrile patients and those unable to provide informed consent. As a result, our incidence calculation may be an under-estimation of the true burden of MDR iNTS disease among the study populations. Different sample sizes across sampled locations in respective countries may limit a direct comparison on AMR occurrence between regions, and thus adjusted incidence estimations may be more suitable for data comparability. The substantial burden of MDR iNTS disease in infants and young children in these countries is likely to undermine the 
effectiveness of therapy, which may lead to prolonged illness and increased risks to case fatalities. For instance, a hospital-based study of iNTS disease in Malawian children exhibited high prevalence of MDR iNTS disease and identified significant risk factors for mortality, including a history of dyspnea, absence of fever, presence of respiratory distress or hypoglycaemia at presentation, and HIV infection [67]. Further studies on MDR and iNTS disease severity are warranted.

\section{Conclusions}

We have found high prevalence of MDR iNTS infections in sub-Saharan African countries which are predominantly caused by $S$. Typhimurium ST313 and $S$. Enteritidis ST11. Our study also provides further insights into the transmissions of MDR ST313 lineage II and the West African Clade of MDR ST11 between West African countries. High incidence of MDR-iNTS disease in children aged $<5$ years is found in several West African countries. Antimicrobial stewardship and continuous surveillance and investigations on the severity and fatalities associated with iNTS disease are instrumental to guide appropriate treatment therapy and enhance control and prevention measures. In addition, the deployment of a safe and effective polyvalent vaccine against the most commonly found iNTS serotypes should be prioritized for the management and prevention of iNTS disease in Africa, particularly in countries where there is high prevalence of MDR iNTS infections, malaria and malnutrition.

\section{Declarations}

\section{Ethics approval and consent to participate}

This research was conducted under the ethical principles of the Declaration of Helsinki. The IVI Institutional Review Board (IRB), the national ethical review committees in each participating country, and local research ethics committees approved the study protocol. All eligible patients meeting the study inclusion criteria were provided with a detailed explanation of the study purpose, and written informed consent was obtained prior to study enrolment. For children, 
written informed consent was obtained from parent or guardian [68].

\section{Consent for publication}

Not applicable.

Availability of data and materials

Raw sequence data are available in the European Nucleotide Archive (projects ERP009684, ERP010763, ERP013866). SMALT version 0.7.4 used is available at:

http://www.sanger.ac.uk/resources/software/smalt/.

\section{Competing interests}

The authors declare no competing interests.

\section{Funding}

This study was supported by the Bill \& Melinda Gates Foundation (grant: OPPGH5231). The findings and conclusions are our own and do not necessarily reflect positions of the Bill \& Melinda Gates Foundation or the US Centers for Disease Control and Prevention. The International Vaccine Institute acknowledges its donors, including the Government of Republic of Korea and the Swedish International Development Cooperation Agency (SIDA). Research infrastructure at the Moshi site was supported by the US National Institutes of Health (R01TW009237; U01 AI062563; R24 TW007988; D43 PA-03-018; U01 AI069484; U01 AI067854; P30 AI064518), and by the UK Biotechnology and Biological Sciences Research Council (BB/J010367). SB is a Sir Henry Dale Fellow, jointly funded by the Wellcome Trust and the Royal Society $(100087 / \mathrm{Z} / 12 / \mathrm{Z})$. DTP is funded as a leadership fellow through the Oak Foundation (OCAY-15-547). 
SEP conducted the phylogenetic, resistance gene and plasmid analyses and interpretation of results under the scientific supervision of DTP. SEP and GDP analysed incidence estimations. SEP structured, drafted, and edited the paper under the scientific guidance from SB and DTP. FM, SEP, VvK, LMCE, UP, GDP, JI, OM, HSG, JAC, RFB, YAS, EOD, RR, ABS, AA, NG, KHK, JM, AGS, PA, HMB, JTH, JMM, LC, BO, BF, NS, TJLR, TMR, LPK, ES, MT, BY, MAET, AS, RK, DMD, AJ, AT, AN, MBA, SVL, JFD, JKP, and FK contributed to data acquisition in the field, interpretation of results, and editing of the paper. All authors read and approved the final draft.

\section{Acknowledgements}

We would like to acknowledge and thank all staff and partners involved in obtaining and processing the data and samples including healthcare facility and laboratory staff at the TSAP network countries. We also thank the WTSI Pathogen Informatics team for help with whole genome sequencing.

\section{References}

1. Morpeth SC, Ramadhani HO, Crump JA. Invasive non-Typhi Salmonella disease in Africa. Clin Infect Dis. 2009;49: 606-11. doi:10.1086/603553

2. Feasey NA, Dougan G, Kingsley RA, Heyderman RS, Gordon MA. Invasive nontyphoidal salmonella disease: an emerging and neglected tropical disease in Africa. Lancet. 2012;379: 2489-2499. doi:10.1016/S0140-6736(11)61752-2

3. Ao TT, Feasey NA, Gordon MA, Keddy KH, Angulo FJ, Crump JA. Global Burden of Invasive Nontyphoidal Salmonella Disease, 2010. Emerg Infect Dis. 2015;21. doi:10.3201/eid2106.140999

4. Gordon MA. Invasive Non-typhoidal Salmonella Disease - epidemiology, pathogenesis and diagnosis. Curr Opin Infect Dis. 2012;24: 484-489. doi:10.1097/QCO.0b013e32834a9980 
5. Enwere G, Biney E, Cheung Y, Zaman SMA, Okoko B, Oluwalana C, et al. Epidemiologic and clinical characteristics of community-acquired invasive bacterial infections in children aged 2-29 months in The Gambia. Pediatr Infect Dis J. 2006;25: 700-705. doi:10.1097/01.inf.0000226839.30925.a5

6. Berkley JA, Lowe BS, Mwangi I, Williams T, Bauni E, Mwarumba S, et al. Bacteremia among Children Admitted to a Rural Hospital in Kenya. N Engl J Med. 2005;352: 39-47. doi:10.1056/NEJMoa040275

7. Sigaúque B, Roca A, Mandomando I, Morais L, Quintó L, Sacarlal J, et al. Communityacquired bacteremia among children admitted to a rural hospital in Mozambique. Pediatr Infect Dis J. 2009;28: 108-113. doi:10.1097/INF.0b013e318187a87d

8. MacLennan CA, Gondwe EN, Msefula CL, Kingsley RA, Thomson NR, White SA, et al. The neglected role of antibody in protection against bacteremia caused by nontyphoidal strains of Salmonella in African children. J Clin Invest. 2008;118: 1553-1562. doi:10.1172/JCI33998

9. Marks F, Kalckreuth V Von, Aaby P, Adu-sarkodie Y, Ahmed M, Tayeb E, et al. Incidence of invasive salmonella disease in sub-Saharan Africa: a multicentre population-based surveillance study. Lancet Glob Heal. 2017;5(3): e310-e323.

10. Okoro CK, Kingsley RA, Connor TR, Harris SR, Parry CM, Al-Mashhadani MN, et al. Intra-continental spread of human invasive Salmonella Typhimurium pathovariants in sub-Saharan Africa. Nat Genet. 2012;44: 1215-1221. doi:10.1038/ng.2423

11. Leekitcharoenphon P, Friis C, Zankari E, Svendsen CA, Price LB, Rahmani M, et al. Genomics of an emerging clone of Salmonella serovar Typhimurium ST313 from Nigeria and the Democratic Republic of Congo. J Infect Dev Ctries. 2013;7: 696-706. doi: $10.3855 /$ jidc. 3328

12. Feasey NA, Cain AK, Msefula CL, Derek P, Maaike A, Aslett M, et al. Drug Resistance in Salmonella enterica ser. Typhimurium Bloodstream Infection, Malawi. Emerg Infect Dis. 2014;20: 1957-1959. doi:10.3201/eid2011.141175 
13. Kariuki S, Gordon MA, Feasey N, Parry CM. Antimicrobial resistance and management of invasive Salmonella disease. Vaccine. 2015;33: C21-9.

doi:10.1016/j.vaccine.2015.03.102.Antimicrobial

14. Okoro CK, Kingsley RA, Connor TR, Harris SR, Parry CM, Al-Mashhadani MN, et al. Intracontinental spread of human invasive Salmonella Typhimurium pathovariants in sub-Saharan Africa. Nat Genet. 2012;44: 1215-1221. doi:10.1038/ng.2423

15. Feasey NA, Hadfield J, Keddy KH, Dallman TJ. Distinct Salmonella Enteritidis lineages associated with enterocolitis in high-income settings and invasive disease in low-income settings. Nat Genet. 2016;48: 1211-1217. doi:10.1038/ng.3644

16. Feasey NA, Archer BN, Heyderman RS, Sooka A, Dennis B, Gordon MA, et al. Typhoid fever and invasive nontyphoid salmonellosis, Malawi and South Africa. Emerg Infect Dis. 2010;16: 1448-1451. doi:10.3201/eid1609.100125

17. Beyene G, Nair S, Asrat D, Mengistu Y, Engers H, Wain J. Multidrug resistant Salmonella Concord is a major cause of salmonellosis in children in Ethiopia. J Infect Dev Ctries. 2011;5: 23-33.

18. Tennant SM, Diallo S, Levy H, Livio S, Sow SO, Tapia M, et al. Identification by PCR of non-typhoidal Salmonella enterica serovars associated with invasive infections among febrile patients in Mali. PLoS Negl Trop Dis. 2010;4: e621. doi:10.1371/journal.pntd.0000621

19. Kariuki S, Gordon MA, Feasey NA, Parry CM, Trust W, Campus G, et al. Antimicrobial resistance and management of invasive Salmonella disease. Vaccine. 2015;33: C21-9. doi:10.1016/j.vaccine.2015.03.102.Antimicrobial

20. Gordon MA, Graham SM, Walsh AL, Wilson L, Phiri A, Molyneux E, et al. Epidemics of invasive Salmonella enterica serovar enteritidis and $S$. enterica Serovar typhimurium infection associated with multidrug resistance among adults and children in Malawi. Clin Infect Dis. 2008;46: 963-9. doi:10.1086/529146

21. Musicha P, Cornick JE, Bar-Zeev N, French N, Masesa C, Denis B, et al. Trends in 
antimicrobial resistance in bloodstream infection isolates at a large urban hospital in Malawi (1998-2016): a surveillance study. Lancet Infect Dis. 2017;17: 1042-1052. doi:10.1016/S1473-3099(17)30394-8

22. Molyneux EM, Mankhambo LA, Ajib P, Graham SM, Forsyth H, Amos P, et al. The outcome of non-typhoidal salmonella meningitis in Malawian children, 1997-2006. Ann Trop Paediatr. 2009;29. doi:10.1179/146532809X401980

23. Kariuki S, Okoro C, Kiiru J, Njoroge S, Omuse G, Langridge G, et al. Ceftriaxoneresistant Salmonella enterica serotype typhimurium sequence type 313 from Kenyan patients is associated with the blaCTX-M-15 gene on a novel IncHI2 plasmid. Antimicrob Agents Chemother. 2015;59: 3133-3139. doi:10.1128/AAC.00078-15

24. Oneko M, Kariuki S, Muturi-Kioi V, Otieno K, Otieno VO, Williamson JM, et al. Emergence of Community-Acquired, Multidrug-Resistant Invasive Nontyphoidal Salmonella Disease in Rural Western Kenya, 2009-2013. Clin Infect Dis. 2015;61: S310-S316. doi:10.1093/cid/civ674

25. Akullian A, Montgomery JM, John-Stewart G, Miller SI, Hayden HS, Radey MC, et al. Multi-drug resistant non-typhoidal Salmonella associated with invasive disease in western Kenya. PLoS Negl Trop Dis. 2018;12: e0006156.

doi:10.1371/journal.pntd.00061566156

26. Nielsen MV, Sarpong N, Krumkamp R, Dekker D, Loag W, Amemasor S, et al. Incidence and characteristics of bacteremia among children in rural Ghana. PLoS One. 2012;7: e44063. doi:10.1371/journal.pone.0044063

27. Kalonji LM, Post A, Phoba MF, Falay D, Ngbonda D, Muyembe JJ, et al. Invasive salmonella infections at multiple surveillance sites in the Democratic Republic of the Congo, 2011-2014. Clin Infect Dis. 2015;61: S346-S353. doi:10.1093/cid/civ713

28. Mandomando I, Jaintilal D, Pons MJ, Vallès X, Espasa M, Mensa L, et al. Antimicrobial susceptibility and mechanisms of resistance in Shigella and Salmonella isolates from children under five years of age with diarrhea in rural Mozambique. Antimicrob Agents 
Chemother. 2009;53: 2450-2454. doi:10.1128/AAC.01282-08

29. Blomberg B, Manji KP, Urassa WK, Tamim BS, Mwakagile DS, Jureen R, et al. Antimicrobial resistance predicts death in Tanzanian children with bloodstream infections: a prospective cohort study. BMC Infect Dis. 2007;7: 1-14. doi:10.1186/1471-2334-7-43

30. Obaro SK, Hassan-Hanga F, Olateju EK, Umoru D, Lawson L, Olanipekun G, et al. Salmonella bacteremia among children in central and Northwest Nigeria, 2008-2015. Clin Infect Dis. 2015;61: S325-S331. doi:10.1093/cid/civ745

31. Van Puyvelde S, Pickard D, Vandelannoote K, Heinz E, Barbé B, de Block T, et al. An African Salmonella Typhimurium ST313 sublineage with extensive drug-resistance and signatures of host adaptation. Nat Commun. 2019;10: 1-12. doi:10.1038/s41467-019$11844-\mathrm{z}$

32. Von Kalckreuth V, Konings F, Aaby P, Adu-Sarkodie Y, Ali M, Aseffa A, et al. The Typhoid Fever Surveillance in Africa Program (TSAP): Clinical, Diagnostic, and Epidemiological Methodologies. Clin Infect Dis. 2016;62: s9-s16. doi:10.1093/cid/civ693

33. Park SE, Pak GD, Aaby P, Adu-Sarkodie Y, Ali M, Aseffa A, et al. The Relationship Between Invasive Nontyphoidal Salmonella Disease, Other Bacterial Bloodstream Infections, and Malaria in Sub-Saharan Africa. Clin Infect Dis. 2016;62: s23-s31. doi:10.1093/cid/civ893

34. Msefula CL, Kingsley RA, Gordon MA, Molyneux E, Molyneux ME, MacLennan CA, et al. Genotypic homogeneity of multidrug resistant $S$. Typhimurium infecting distinct adult and childhood susceptibility groups in Blantyre, Malawi. PLoS One. 2012;7: e42085. doi:10.1371/journal.pone.0042085

35. Zerbino DR, Birney E. Velvet: Algorithms for de novo short read assembly using de Bruijn graphs. Genome Res. 2008;18: 821-829. doi:10.1101/gr.074492.107

36. Gladman S, Seemann T. Velvet optimiser, https://github.com/Victorian-Bioinformatics- 
Consortium/VelvetOptimiser.git. Available:

http://www.bioinformatics.net.au/software.velvetoptimiser.shtml

37. Page AJ, Taylor B, Delaney AJ, Soares J, Seemann T, Keane JA, et al. SNP-sites: rapid efficient extraction of SNPs from multi-FASTA alignments. Microb Genomics. 2016;2: e000056. doi:10.1099/mgen.0.000056

38. Seemann T. Prokka: Rapid prokaryotic genome annotation. Bioinformatics. 2014;30: 2068-2069. doi:10.1093/bioinformatics/btu153

39. Page AJ, Cummins CA, Hunt M, Wong VK, Reuter S, Holden MTG, et al. Roary: Rapid large-scale prokaryote pan genome analysis. Bioinformatics. 2015;31: 3691-3693. doi:10.1093/bioinformatics/btv421

40. Li H, Handsaker B, Wysoker A, Fennell T, Ruan J, Homer N, et al. The Sequence Alignment/Map format and SAMtools. Bioinformatics. 2009;25: 2078-2079. doi:10.1093/bioinformatics/btp352

41. Kurtz S, Phillippy A, Delcher A, Smoot M, Shumway M, Antonescu C, et al. Versatile and open software for comparing large genomes. Genome Biol. 2004;5: R12. doi:10.1186/gb-2004-5-2-r12

42. Croucher NJ, Page AJ, Connor TR, Delaney AJ, Keane JA, Bentley SD, et al. Rapid phylogenetic analysis of large samples of recombinant bacterial whole genome sequences using Gubbins. Nucleic Acids Res. 2015;43: e15. doi:10.1093/nar/gku1 196

43. Stamatakis A. RAxML version 8: A tool for phylogenetic analysis and post-analysis of large phylogenies. Bioinformatics. 2014;30: 1312-1313.

doi:10.1093/bioinformatics/btu033

44. Letunic I, Bork P. Interactive Tree Of Life (iTOL): An online tool for phylogenetic tree display and annotation. Bioinformatics. 2007;23: 127-128.

doi:10.1093/bioinformatics/bt1529

45. Inouye M, Dashnow H, Raven L-A, Schultz MB, Pope BJ, Tomita T, et al. SRST2: Rapid genomic surveillance for public health and hospital microbiology labs. Genome 
Med. 2014;6: 90. doi:10.1186/s13073-014-0090-6

46. Gupta SK, Padmanabhan BR, Diene SM, Lopez-Rojas R, Kempf M, Landraud L, et al. ARG-annot, a new bioinformatic tool to discover antibiotic resistance genes in bacterial genomes. Antimicrob Agents Chemother. 2014;58: 212-220. doi:10.1128/AAC.0131013

47. Carattoli A, Zankari E, Garcia-Fernandez A, Larsen MV, Lund O, Villa L, et al. In Silico Detection and Typing of Plasmids using PlasmidFinder and Plasmid Multilocus Sequence Typing. Antimicrob Agents Chemother. 2014;58: 3895-3903. doi:10.1128/AAC.02412-14

48. Achtman M, Wain J, Weill FX, Nair S, Zhou Z, Sangal V, et al. Multilocus sequence typing as a replacement for serotyping in Salmonella enterica. PLoS Pathog. 2012;8: e1002776. doi:10.1371/journal.ppat.1002776

49. Zhang S, Yin Y, Jones MB, Zhang Z, Kaiser BLD, Dinsmore BA, et al. Salmonella serotype determination utilizing high-throughput genome sequencing data. J Clin Microbiol. 2015;53: 1685-1692. doi:10.1128/JCM.00323-15

50. Bankevich A, Nurk S, Antipov D, Gurevich AA, Dvorkin M, Kulikov AS, et al. SPAdes: a new genome assembly algorithm and its applications to single-cell sequencing. J Comput Biol. 2012;19: 455-477. doi:10.1089/cmb.2012.0021

51. Wick RR, Schultz MB, Zobel J, Holt KE. Bandage: Interactive visualization of de novo genome assemblies. Bioinformatics. 2015;31: 3350-3352. doi:10.1093/bioinformatics/btv383

52. Panzner U, Pak GD, Aaby P, Adu-Sarkodie Y, Ali M, Aseffa A, et al. Utilization of Healthcare in the Typhoid Fever Surveillance in Africa Program. Clin Infect Dis. 2016;62: S56-S68. doi:10.1093/cid/civ891

53. Jajere SM. A review of Salmonella enterica with particular focus on the pathogenicity and virulence factors, host specificity and antimicrobial resistance including multidrug resistance. Vet World. 2019;12: 504-521. doi:10.14202/vetworld.2019.504-521 
54. Sow AG, Wane AA, Diallo MH, Boye CSB, Ä̈dara-Kane A. Genotypic characterization of antibiotic-resistant Salmonella Enteritidis isolates in Dakar, Senegal. J Infect Dev Ctries. 2007;1: 284-288.

55. Keddy KH, Takuva S, Musekiwa A, Puren AJ, Sooka A, Karstaedt A, et al. An association between decreasing incidence of invasive non-typhoidal salmonellosis and increased use of antiretroviral therapy, Gauteng Province, South Africa, 2003-2013. PLoS One. 2017;12: e0173091. doi:10.1371/journal.pone.0173091

56. Tennant SM, MacLennan CA, Simon R, Martin LB, Khan MI. Nontyphoidal salmonella disease: Current status of vaccine research and development. Vaccine. 2016;34: 29072910. doi:10.1016/j.vaccine.2016.03.072

57. International Vaccine Institute. IVI to Accelerate Efforts in iNTS Vaccine Development - IVI. [cited 6 Mar 2020]. Available: https://www.ivi.int/ivi-to-accelerate-efforts-in-intsvaccine-development/

58. Rodríguez I, Rodicio MR, Guerra B, Hopkins KL. Potential International Spread of Multidrug-Resistant Invasive Salmonella enterica Serovar Enteritidis. Emerg Infect Dis. 2012;18: 1173-6. doi:10.3201/eid1807.120063

59. Kariuki S, Revathi G, Kariuki N, Kiiru J, Mwituria J, Muyodi J, et al. Invasive multidrug-resistant non-typhoidal Salmonella infections in Africa: Zoonotic or anthroponotic transmission? J Med Microbiol. 2006;55: 585-591. doi:10.1099/jmm.0.46375-0

60. Post AS, Diallo SN, Guiraud I, Lompo P, Tahita MC, Maltha J, et al. Supporting evidence for a human reservoir of invasive non-Typhoidal Salmonella from household samples in Burkina Faso. PLoS Negl Trop Dis. 2019;13: 1-18. doi:10.1371/journal.pntd.0007782

61. Kingsley RA, Msefula CL, Thomson NR, Kariuki S, Holt KE, Gordon MA, et al. Epidemic multiple drug resistant Salmonella Typhimurium causing invasive disease in sub-Saharan Africa have a distinct genotype. Genome Res. 2009;19: 2279-2287. 
doi:10.1101/gr.091017.109

62. Akinyemi KO, Bamiro BS, Coker AO. Salmonellosis in Lagos, Nigeria: Incidence Coinfection, Patterns of Antimicrobial Resistance, and Emergence of Reduced Susceptibility to Fluoroquinolones. J Heal Popul Nutr. 2007;25: 351-358.

63. Harrois D, Breurec S, Seck A, Delauné A, Le Hello S, Pardos de la Gándara M, et al. Prevalence and characterization of extended-spectrum $\beta$-lactamase-producing clinical Salmonella enterica isolates in Dakar, Senegal, from 1999 to 2009. Clin Microbiol Infect. 2014;20. doi:10.1111/1469-0691.12339

64. Lunguya O, Lejon V, Phoba M-F, Bertrand S, Vanhoof R, Glupczynski Y, et al. Antimicrobial Resistance in Invasive Non-typhoid Salmonella from the Democratic Republic of the Congo: Emergence of Decreased Fluoroquinolone Susceptibility and Extended-spectrum Beta Lactamases. PLoS Negl Trop Dis. 2013;7: e2103. doi:10.1371/journal.pntd.0002103

65. Keddy KH, Musekiwa A, Sooka A, Karstaedt A, Nana T, Seetharam S, et al. Clinical and microbiological features of invasive nontyphoidal Salmonella associated with HIVinfected patients, Gauteng Province, South Africa. Med (United States). 2017;96: 1-7. doi:10.1097/MD.0000000000006448

66. Park SE, Pham DT, Boinett C, Wong VK, Pak GD, Panzner U, et al. The phylogeography and incidence of multi-drug resistant typhoid fever in sub-Saharan Africa. Nat Commun. 2018;9: 5094. doi:10.1038/s41467-018-07370-z

67. MacLennan CA, Msefula CL, Gondwe EN, Gilchrist JJ, Pensulo P, Mandala WL, et al. Presentation of life-threatening invasive nontyphoidal Salmonella disease in Malawian children: A prospective observational study. PLoS Negl Trop Dis. 2017;11: 1-16. doi:10.1371/journal.pntd.0006027

68. Von Kalckreuth V, Konings F, Aaby P, Adu-Sarkodie Y, Ali M, Aseffa A, et al. The Typhoid Fever Surveillance in Africa Program (TSAP): Clinical, Diagnostic, and Epidemiological Methodologies. Clin Infect Dis. 2016;62: s9-s16. 
doi:10.1093/cid/civ693.

\section{Figure Legends}

Figure 1. The geographical distribution of iNTS genotypes and serovars in the sampled countries in sub-Saharan Africa.

Different colours in the pie charts correspond to different sequence types and serovars of iNTS isolates in our study sites. The size of the pie charts corresponds to the numbers of isolates in each country. Countries highlighted in red and grey are with and without MDR iNTS isolates respectively.

Figure 2. Antimicrobial resistant genes and plasmids associated with iNTS isolates circulating in sampled sub-Saharan Africa countries.

Maximum likelihood phylogenetic tree based on the core genes of all our iNTS isolates and their corresponding metadata. First column shows the sequence types in different colour. Second column corresponds to the countries where our iNTS isolates were detected. The remaining columns exhibit a heatmap of detected AMR genes and plasmid replicons. The tree scale bar indicates the number of substitutions per variable site.

Figure 3. Novel IncI1 virulence-resistance plasmid (pSEP) in an S. Enteritidis ST11 isolate. Plasmid comparison analyses between the novel virulence-resistance IncI1 plasmid pSEP (bottom) and the reference virulence IncF plasmid pSENV (top). The grey blocks show the BLASTn comparison between the two plasmids. Some annotations are added for both plasmids. Red coloured arrows are genes associated with virulence and AMR. Blue coloured arrows are genes associated with conjugation. Grey coloured arrows correspond to plasmid replication and stability. Green coloured genes are associated with transposon elements. 


\section{Figure 4. The phylogeography of $S$. Typhimurium ST313 lineage II and $S$. Enteritidis}

\section{ST11 in sub-Saharan Africa.}

a) Maximum likelihood phylogenetic tree of my $S$. Typhimurium-ST313 lineage II isolates in the context of sub-Saharan Africa continent. Red circles at the terminal leaves correspond to our study isolates. First column shows different colour-coded countries from where all analysed isolates originate. Second column shows MDR and non-MDR isolates in red and grey respectively. b) Maximum likelihood phylogenetic tree of my $S$. Enteritidis-ST11 isolates in the global context of $S$. Enteritidis. Red circles at the terminal leaves correspond to our study isolates. First and second columns show regions and resistant phenotypes in different colours. The tree scales indicate the number of substitutions per variable site. 


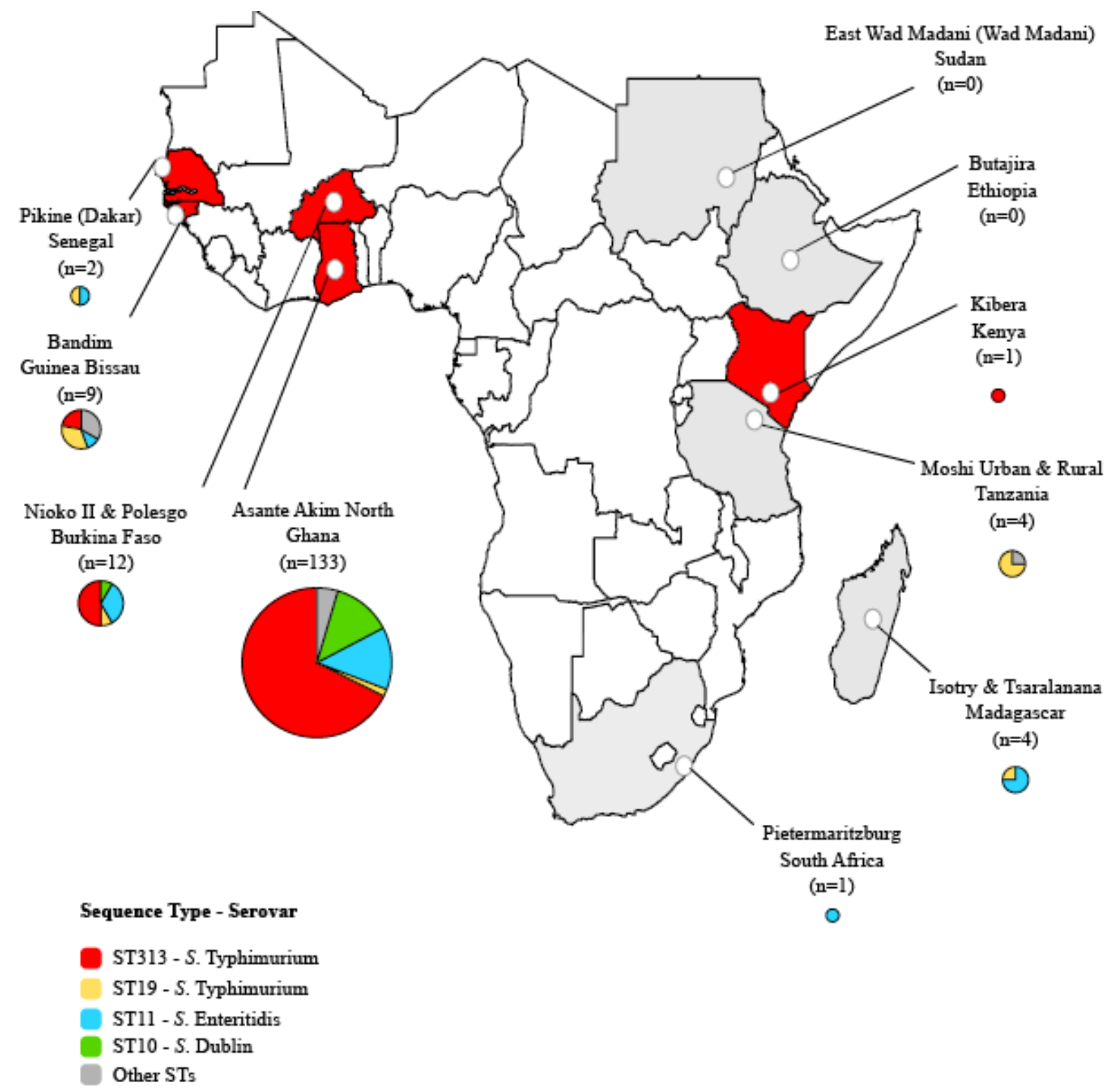

Figure 1. The geographical distribution of iNTS genotypes and serovars in the sampled countries in sub-Saharan Africa

Different colours in the pie charts correspond to different sequence types and serovars of iNTS isolates in our study sites. The size of the pie charts corresponds to the numbers of isolates in each country. Countries highlighted in red and grey are with and without MDR iNTS isolates respectively. 


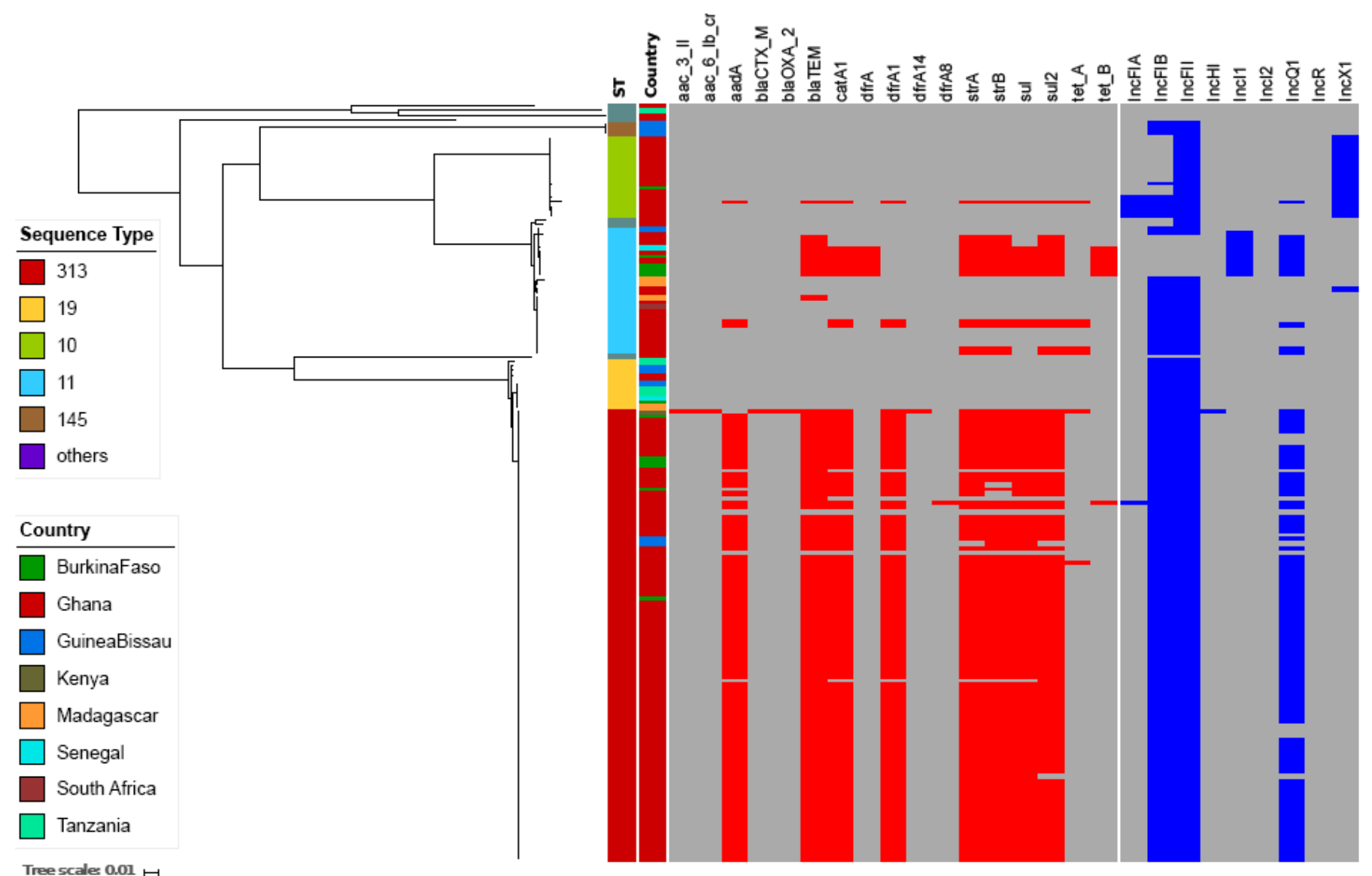

Figure 2. Antimicrobial resistant genes and plasmids associated with iNTS isolates circulating in sampled sub-Saharan Africa countries

Maximum likelihood phylogenetic tree based on the core genes of all our iNTS isolates and their corresponding metadata. First column shows the sequence types in different colour. Second column corresponds to the countries where our iNTS isolates were detected. The remaining columns exhibit a heatmap of detected AMR genes and plasmid replicons. The tree scale bar indicates the number of substitutions per variable site. 


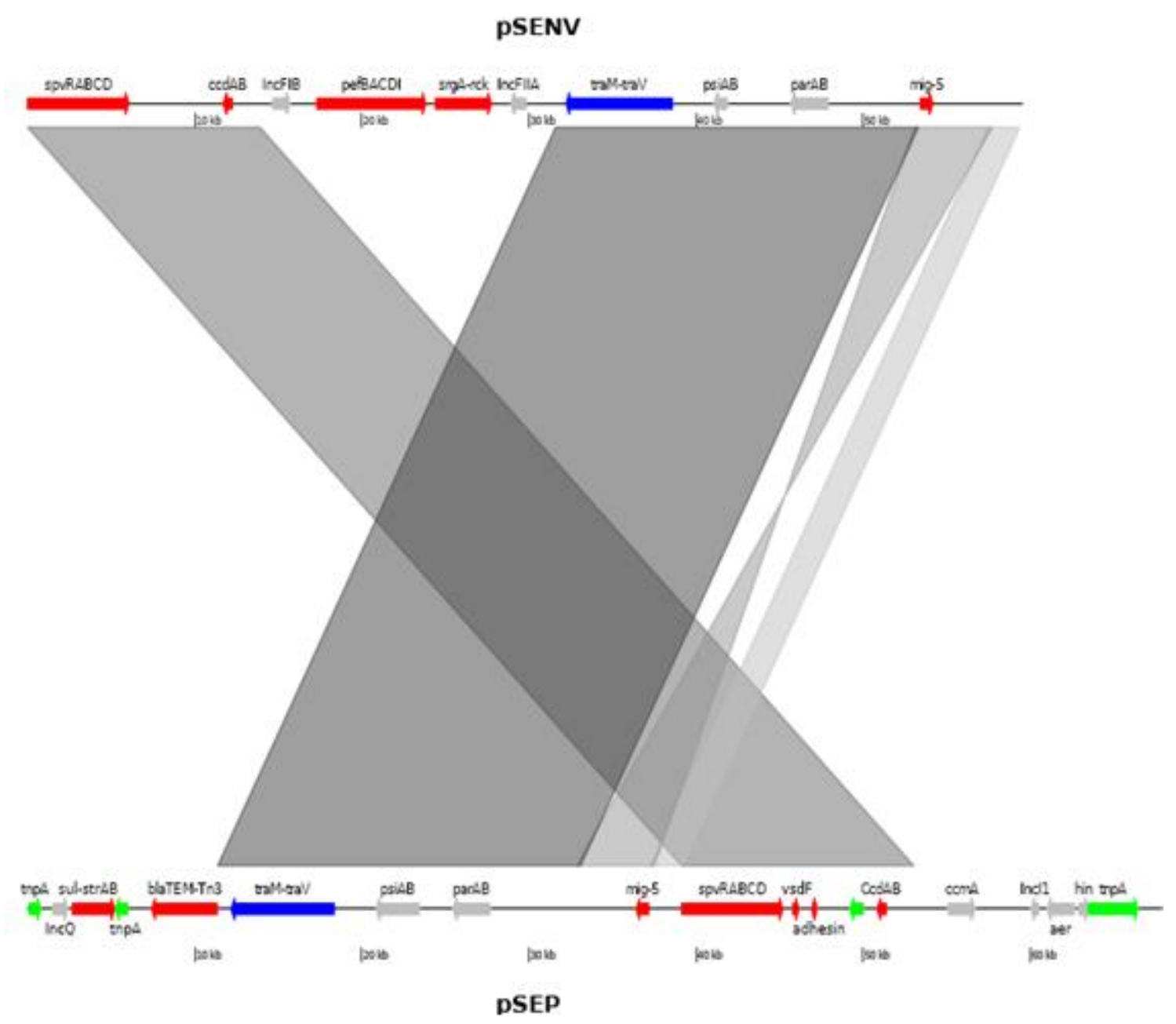

Figure 3. Novel IncI1 virulence-resistance plasmid (pSEP) in an S. Enteritidis ST11 isolate

Plasmid comparison analyses between the novel virulence-resistance IncI1 plasmid pSEP (bottom) and the reference virulence IncF plasmid pSENV (top). The grey blocks show the BLASTn comparison between the two plasmids. Some annotations are added for both plasmids. Red coloured arrows are genes associated with virulence and AMR. Blue coloured arrows are genes associated with conjugation. Grey coloured arrows correspond to plasmid replication and stability. Green coloured genes are associated with transposon elements. 
4a. S. Typhimurium ST313 Lineage II

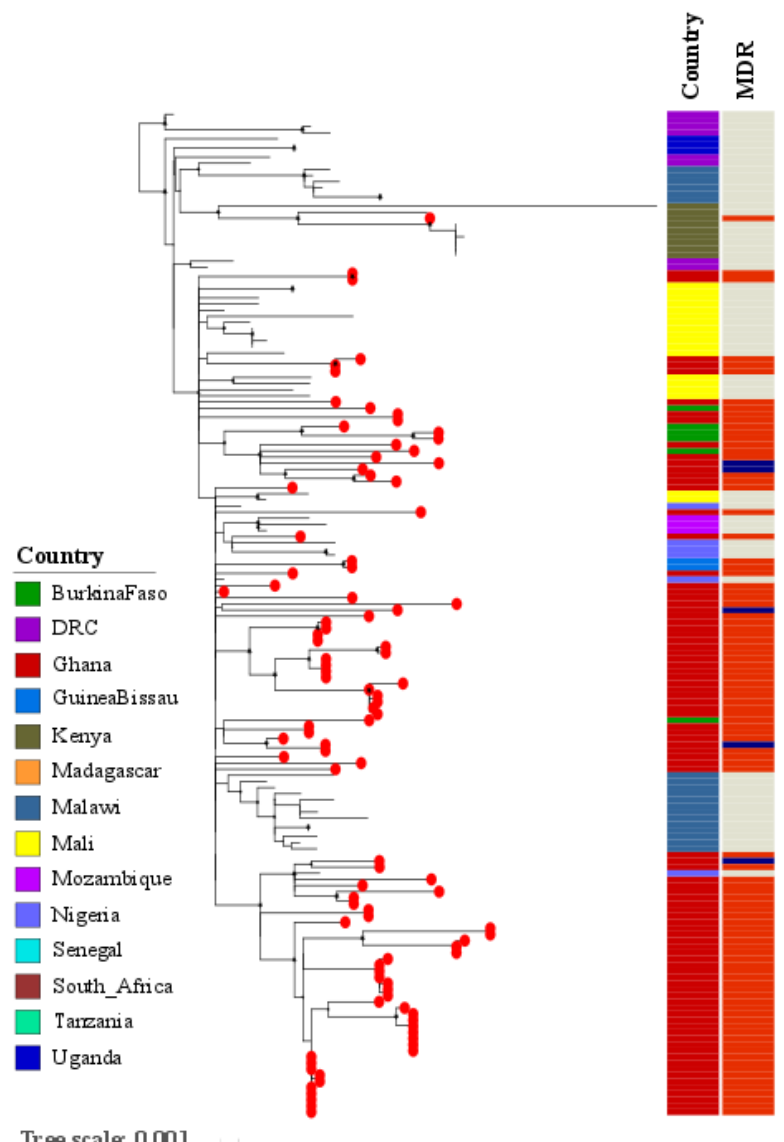

4b. S. Enteritidis STI1

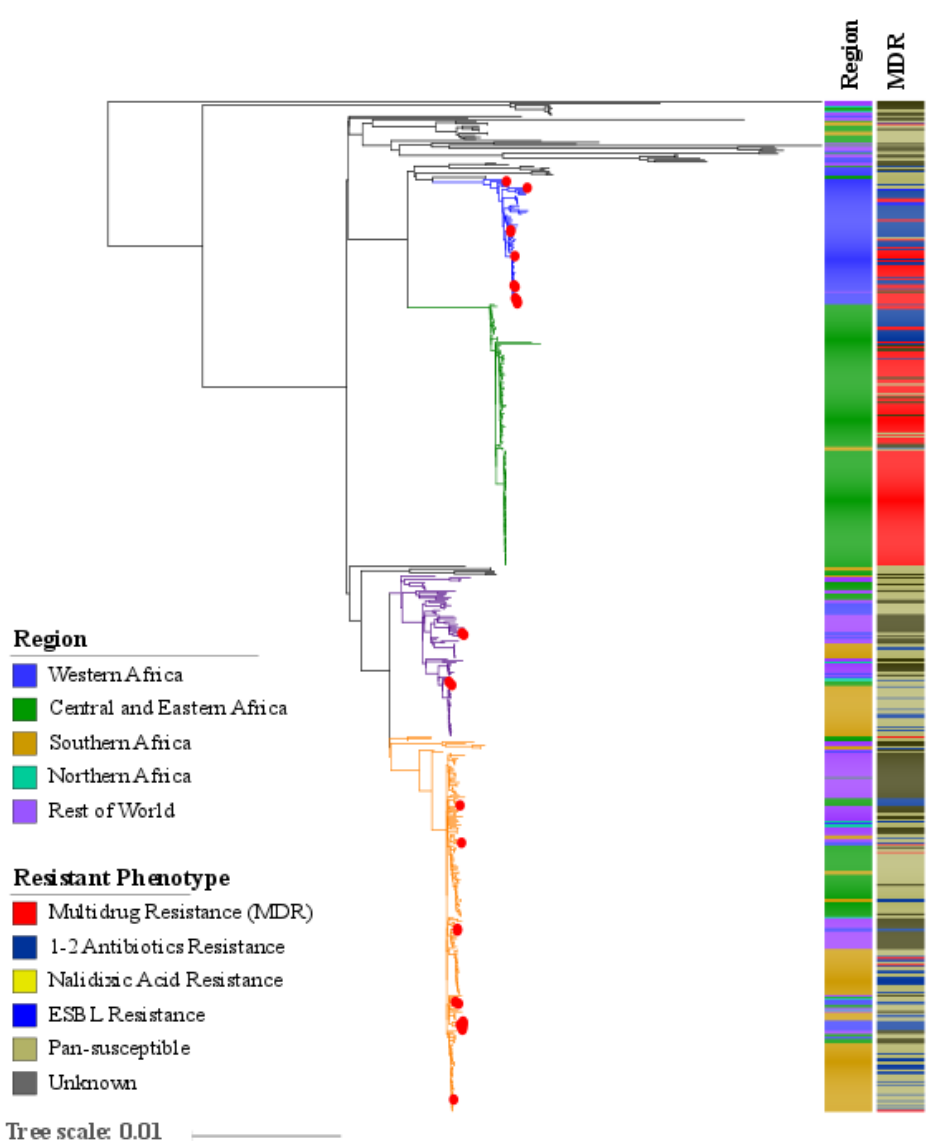

Tree scales 0.01

Figure 4. The phylogeography of $S$. Typhimurium ST313 lineage II and $S$. Enteritidis ST11 in sub-Saharan Africa

a) Maximum likelihood phylogenetic tree of my S. Typhimurium-ST313 lineage II isolates in the context of sub-Saharan Africa continent. Red circles at 
the terminal leaves correspond to our study isolates. First column shows different colour-coded countries from where all analysed isolates originate.

Second column shows MDR and non-MDR isolates in red and grey respectively. b) Maximum likelihood phylogenetic tree of our S. Enteritidis-ST11

isolates in the global context of $S$. Enteritidis. Red circles at the terminal leaves correspond to our study isolates. First and second columns show regions and resistant phenotypes in different colours. The tree scales indicate the number of substitutions per variable site. 
Table 1. The distribution of iNTS serovars and genotypes circulating in the sampled countries in sub-Saharan Africa

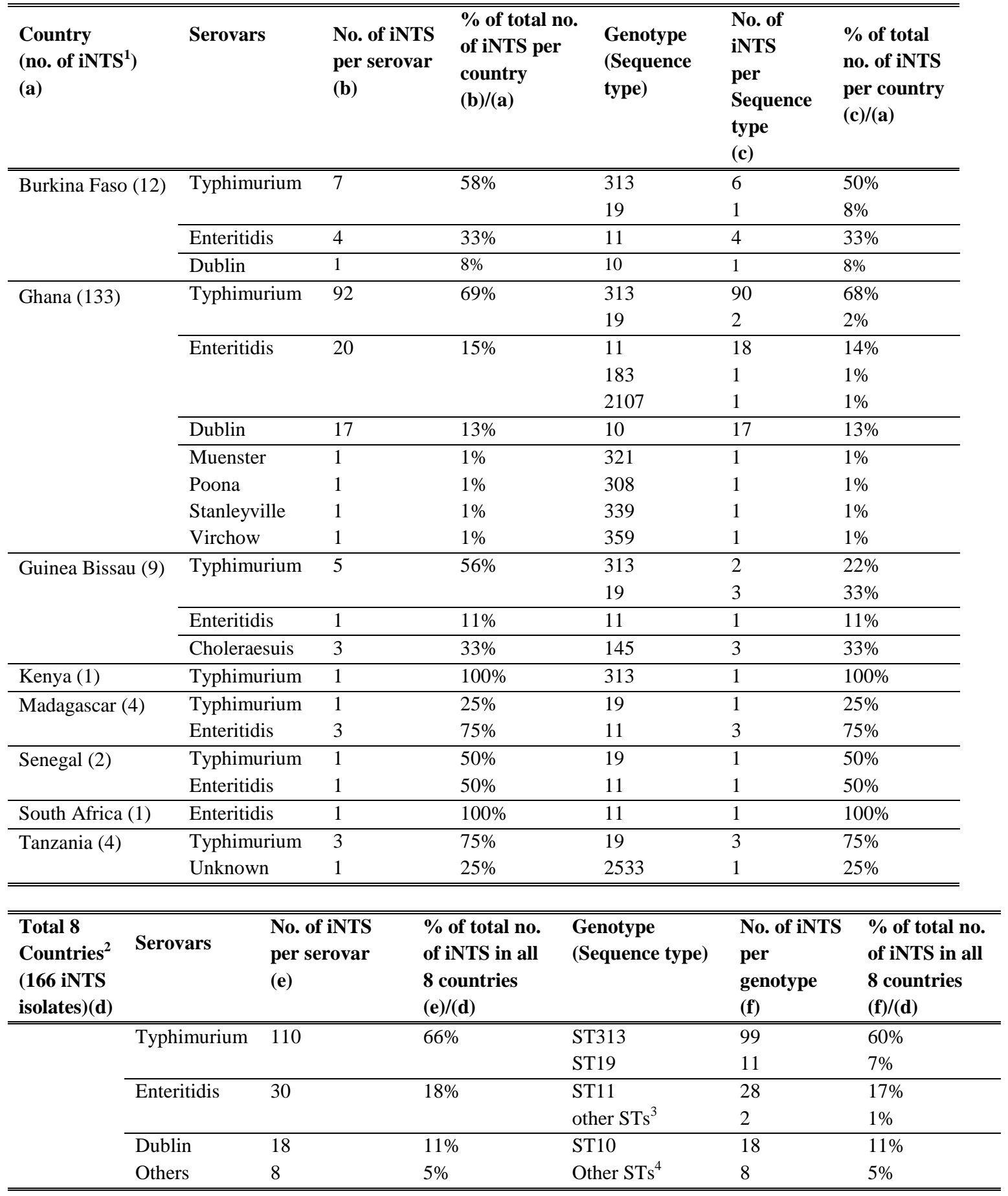

\footnotetext{
${ }^{1}$ iNTS: invasive nontyphoidal Salmonella
}

${ }^{2}$ Total 8 countries: Burkina Faso, Ghana, Guinea-Bissau, Kenya, Madagascar, Senegal, South Africa, and Tanzania presented in this table. No iNTS isolates were yielded in Sudan and Ethiopia.

${ }^{3}$ Other sequence types of $S$. Enteritidis detected: 1 ST183 (isolate yielded from Ghana; age/sex unknown due to missing data) and 1 ST2107 (from Ghana; a 22 year old female); both non-MDR and no antimicrobial resistant genes detected.

${ }^{4}$ Sequence types of the other NTS serovars: 1 Muenster ST321 (yielded from Ghana; age/sex/year unknown due to missing data), 1 Poona ST308 (yielded from Ghana in 2008; age/sex unknown due to missing data), 1 Stanleyville ST339 (yielded from Ghana; age/sex/year unknown due to missing data), 1 Virchow ST359 (from Ghana; age/sex/year unknown due to missing data) 3 Choleraesui ST145 (2 isolates yielded from 1 year old female infants in 2010 and 2011 and 1 from 3 year old female infant in 2012; all from Guinea-Bissau), 1 Unknown ST2533 from Tanzania. 
Table 2. The distribution of MDR iNTS and gyrA mutation (fluoroquinolone resistance) in the sampled countries in sub-Saharan Africa

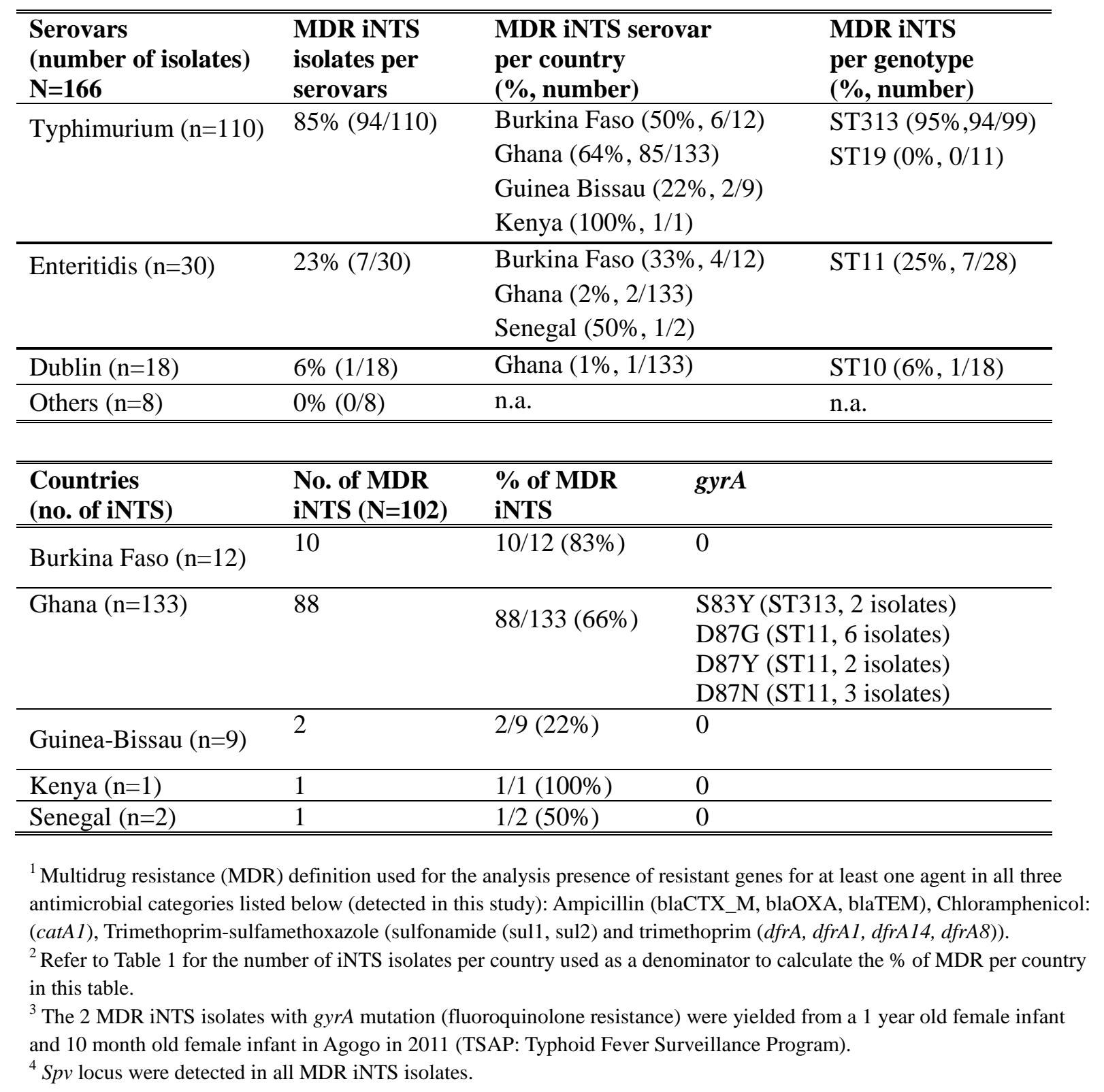


Table 3. Incidence estimates of MDR iNTS disease in sub-Saharan Africa

\begin{tabular}{|c|c|c|c|c|c|c|c|c|c|c|c|}
\hline \multirow[t]{2}{*}{ Country $^{1}$} & \multirow{2}{*}{$\begin{array}{r}\text { Age group } \\
\text { in years }\end{array}$} & \multicolumn{4}{|c|}{ 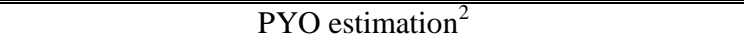 } & \multirow{2}{*}{$\begin{array}{l}\text { Recruitment } \\
\text { proportion }^{3}\end{array}$} & \multirow{2}{*}{$\begin{array}{l}\text { Genome- } \\
\text { sequenced } \\
\text { iNTS cases }\end{array}$} & \multirow{2}{*}{$\begin{array}{l}\text { Crude } \\
\text { MDR } \\
\text { iNTS } \\
\text { cases }\end{array}$} & \multirow{2}{*}{$\begin{array}{r}\text { Crude } \\
\text { MDR } \\
\text { iNTS } \\
\text { incidence } \\
\text { per } \\
100,000 \\
\text { PYO }\end{array}$} & \multirow{2}{*}{$\begin{array}{r}\text { Adjusted } \\
\text { MDR } \\
\text { iNTS } \\
\text { cases }\end{array}$} & \multirow{2}{*}{$\begin{array}{r}\text { Adjusted MDR } \\
\text { iNTS incidence } \\
\text { per 100,000 PYO } \\
(95 \% \mathrm{CI})^{4}\end{array}$} \\
\hline & & $\begin{array}{r}\text { Proportion of } \\
\text { catchment } \\
\text { population } \\
\text { visiting study } \\
\text { facility in case of } \\
\text { fever }(95 \% \mathrm{CI})\end{array}$ & $\begin{array}{l}\text { Catchment } \\
\text { population }\end{array}$ & $\begin{array}{r}\text { Catchment } \\
\text { population } \\
\text { adjusted by } \\
\text { health- } \\
\text { seeking } \\
\text { behaviour }\end{array}$ & PYO & & & & & & \\
\hline \multicolumn{12}{|l|}{ Burkina Faso } \\
\hline \multirow[t]{6}{*}{ Nioko II } & $0-1$ & $81 \%(74-88)$ & 2208 & 1788 & 2097 & 247/1297 (19\%) & 2 & 1 & 48 & 5 & $251(107-590)$ \\
\hline & $2-4$ & $81 \%(75-86)$ & 1823 & 1477 & 2097 & $235 / 1259(19 \%)$ & 3 & 3 & 143 & 16 & $753(460-1233)$ \\
\hline & $5-14$ & $81 \%(78-84)$ & 4295 & 3479 & 4889 & $228 / 889(26 \%)$ & 2 & 1 & 20 & 4 & $79(29-214)$ \\
\hline & $<15$ & n.a. & 8326 & 6744 & 9083 & n.a. & 7 & 5 & 55 & 25 & 274 (185-406) \\
\hline & $\geq 15$ & $81 \%(79-83)$ & 9428 & 7637 & 10676 & $208 / 759(27 \%)$ & 1 & 1 & 9 & 4 & $35(13-96)$ \\
\hline & All & n.a. & 17754 & 14381 & 19759 & n.a. & 8 & 6 & 30 & 29 & $145(100-209)$ \\
\hline \multirow[t]{6}{*}{ Polesgo } & $0-1$ & $92 \%(86-99)$ & 896 & 824 & 929 & $117 / 475(25 \%)$ & 1 & 1 & 108 & 4 & 431 (162-1147) \\
\hline & $2-4$ & $83 \%(76-89)$ & 856 & 710 & 992 & $148 / 466(32 \%)$ & 2 & 2 & 202 & 6 & $630(288-1380)$ \\
\hline & $5-14$ & $87 \%(83-91)$ & 1734 & 1509 & 2104 & $252 / 510(49 \%)$ & 0 & 0 & 0 & 0 & 0 \\
\hline & $<15$ & & 3486 & 3043 & 4025 & n.a. & 3 & 3 & 75 & 10 & $255(138-470)$ \\
\hline & $\geq 15$ & $87 \%(84-89)$ & 4088 & 3557 & 4917 & $239 / 629(38 \%)$ & 1 & 1 & 20 & 3 & 54 (16-179) \\
\hline & All & n.a. & 7574 & 6600 & 8942 & n.a. & 4 & 4 & 45 & 13 & $144(83-249)$ \\
\hline \multicolumn{12}{|l|}{ Ghana $^{5}$} \\
\hline \multirow[t]{9}{*}{ AAN } & $0-1$ & $16 \%(14-18)$ & 11222 & 1760 & 4080 & $41 \%$ & 88 & 24 & 588 & 59 & 1435 (1110-1854) \\
\hline & $2-4$ & $16 \%(13-18)$ & 8086 & 1268 & 2940 & $41 \%$ & 23 & 9 & 306 & 22 & $747(491-1135)$ \\
\hline & $0-4$ & n.a. & n.a. & n.a. & n.a. & n.a. & 111 & 33 & n.a. & n.a. & n.a. \\
\hline & $5-14$ & $16 \%(15-17)$ & 34439 & 5415 & 12554 & $623 / 1657(38 \%)$ & 6 & 6 & 48 & 16 & $126(77-206)$ \\
\hline & $<15$ & n.a. & 53747 & 8443 & 19574 & n.a. & 117 & 39 & 147 & 81 & $414(333-515)$ \\
\hline & $\geq 15$ & n.a. & n.a. & n.a. & n.a. & n.a. & 16 & 10 & n.a. & n.a. & n.a. \\
\hline & All_TSAP & n.a. & n.a. & n.a. & n.a. & n.a. & 133 & 49 & n.a. & n.a. & n.a. \\
\hline & Non_TSAP $_{6}$ & n.a. & n.a. & n.a. & n.a. & n.a. & 49 & 39 & n.a. & n.a. & n.a. \\
\hline & All & n.a. & n.a. & n.a. & n.a & n.a. & 182 & 88 & n.a. & n.a. & n.a. \\
\hline \multicolumn{12}{|l|}{ Guinea Bissau } \\
\hline \multirow[t]{6}{*}{ Bandim } & $0-1$ & $46 \%(39-54)$ & 10852 & 4992 & 5198 & 206/631 (33\%) & 7 & 2 & 96 & 15 & $291(176-482)$ \\
\hline & $2-4$ & $43 \%(37-48)$ & 7307 & 3142 & 3866 & $175 / 359(49 \%)$ & 1 & 0 & 26 & 2 & $53(13-208)$ \\
\hline & $5-14$ & $42 \%(41-48)$ & 19905 & 8360 & 11101 & $187 / 380(49 \%)$ & 1 & 0 & 18 & 4 & $37(14-97)$ \\
\hline & $<15$ & n.a. & 38064 & 16494 & 20165 & n.a. & 9 & 2 & 40 & 21 & $105(69-161)$ \\
\hline & $\geq 15$ & $45 \%(43-47)$ & 62694 & 28212 & 37109 & $105 / 163(64 \%)$ & 0 & 0 & 0 & 0 & 0 \\
\hline & All & n.a. & 100758 & 44706 & 57274 & n.a. & 9 & 2 & 14 & 21 & $37(24-57)$ \\
\hline
\end{tabular}

Kenya 


\begin{tabular}{|c|c|c|c|c|c|c|c|c|c|c|c|}
\hline \multirow[t]{6}{*}{ Kibera } & $0-1$ & $42 \%(38-47)$ & 3467 & 1456 & 2031 & 99/99 (100\%) & 0 & 0 & 0 & 0 & 0 \\
\hline & $2-4$ & $39 \%(36-43)$ & 3070 & 1197 & 2039 & $312 / 312(100 \%)$ & 0 & 0 & 0 & 0 & 0 \\
\hline & 5-14 & $43 \%(39-47)$ & 7514 & 3231 & 5722 & $539 / 539(100 \%)$ & 0 & 0 & 0 & 0 & 0 \\
\hline & $<15$ & n.a. & 14051 & 5884 & 9792 & n.a. & 0 & 0 & 0 & 0 & 0 \\
\hline & $\geq 15$ & $35 \%(32-38)$ & 15263 & 5342 & 9228 & $301 / 301(100 \%)$ & 1 & 1 & 11 & 1 & $11(2-77)$ \\
\hline & All & n.a. & 29314 & 11227 & 19020 & n.a. & 1 & 1 & 5 & 1 & $5(1-37)$ \\
\hline \multicolumn{12}{|l|}{ Senegal $^{7}$} \\
\hline \multirow[t]{6}{*}{ Pikine } & $0-1$ & $39 \%(32-46)$ & 20120 & 7837 & 11194 & n.a. & 0 & 0 & n.a. & n.a. & n.a. \\
\hline & $2-4$ & $37 \%(33-41)$ & 30180 & 11097 & 15851 & n.a. & 1 & 1 & n.a. & n.a. & n.a. \\
\hline & 5-14 & $31 \%(28-34)$ & 96152 & 29807 & 42577 & n.a. & 0 & 0 & n.a. & n.a. & n.a. \\
\hline & $<15$ & n.a. & 146452 & 48741 & 69623 & n.a. & 1 & 1 & n.a. & n.a. & n.a. \\
\hline & $\geq 15$ & $30 \%(28-31)$ & 195726 & 58718 & 83874 & n.a. & 1 & 0 & n.a. & n.a. & n.a. \\
\hline & All & n.a. & 342178 & 107459 & 153496 & n.a. & 2 & 1 & n.a. & n.a. & n.a. \\
\hline
\end{tabular}

positive iNTS isolates confirmed via whole genome sequencing. MDR iNTS were found in 5/8 countries presented in this table. No MDR iNTS were detected via whole genome sequencing from the isolates yielded from Madagascar, South Africa, and Tanzania.

${ }^{2,3}$ PYO estimation methodologies have been published in detail in the TSAP typhoid burden paper (Marks et al, Lancet Global Health, 2017).

${ }^{4}$ Adjusted MDR iNTS incidence per 100,000 PYO (95\% CI): adjustments for case recruitment and error factors.

${ }^{5}$ Ghana samples included in this estimation of MDR iNTS are from the TSAP only.

${ }^{6}$ Non-TSAP includes other fever surveillance performed in Agogo ("IsolateAgogo", "FISA", and "TYSA"). Refer to the method section.

${ }^{7}$ Adjusted MDR incidence of iNTS per 100,000 PYO could not be estimated for the study site in Senegal due to unavailable data on the recruitment proportion. The one MDR iNTS patient confirmed and presented in this table (Crude MDR iNTS case) was a male infant aged 17 months, infected in 2012 with $S$. Enteritidis ST11. The one non-MDR iNTS patient presented in this table (Genome-sequenced iNTS case) were a male adult aged 66 years, infected in 2012 with $S$. Typhimurium. 